\title{
Optical 3D Methods in Steel Sheet Container Drop Test Analysis
}

\author{
K.-P. Gründer, D. Kadoke, C. Protz, U. Zencker \\ BAM Bundesanstalt für Materialforschung und -prüfung, \\ Unter den Eichen 87, 12205 Berlin, Germany \\ klaus-peter.gruender@bam.de
}

\begin{abstract}
:
In the context of a research project at BAM various drop tests and material as well as component investigations have to be performed with respect to steel sheet container drop test analysis. Both, experimental and computational methods (Finite Element Analysis) are involved. Among electrical sensor techniques optical measurement methods capable of dynamic and static 3D deformation analysis are applied. On the one hand this includes the Projected Fringes Method which provides a virtual digital round-about model of the actual package surface shape. On the other hand there is the Stereo Photogrammetry used for high speed imaging of points rich in contrast and permanently fixed to the container while dropping. Technical features of both methods are discussed. Diverse measurement results are compared with those of alternative experimental techniques as well as numerical simulations.
\end{abstract}

Key words: Steel sheet container, drop test, numerical simulation, optical digitization, high speed stereo photogrammetry

\section{Introduction}

The BAM Federal Institute for Materials Research and Testing is commissioned to approve RAM $^{1)}$ container types for interim storage and final disposal of radioactive waste. The assessment procedure applied by BAM is usually based on a combination of experimental methods and numerical calculations in conjunction with material and component testing. Within a research project, drop tests of a steel sheet container designed for the German KONRAD repository were conducted for validation and further development of numerical simulations of container tests. A reliable Finite Element (FE) model describing the mechanical behaviour of a box-shaped steel sheet container being exposed to different ways of drop loading shall be developed. The present paper concentrates on some aspects of analysing the test container using optical measurement methods.

\section{Drop Tests}

Depending on the level of radioactivity, containers for the KONRAD repository have to meet acceptance criteria with respect to e.g. outer dimensions, gross weight, container material, stacking height, leakage rate, corrosion protection, integrity, ISO standards for container lifting corners, thermal requirements and fire tests. Furthermore, the container assessment procedure includes drop tests from different heights and drop orientations. Within the studies discussed here, drop tests were conducted using KONRAD steel sheet containers of Type $V$ [1] at the BAM Test Site Technical Safety (TTS) next to Berlin (Fig. 1).

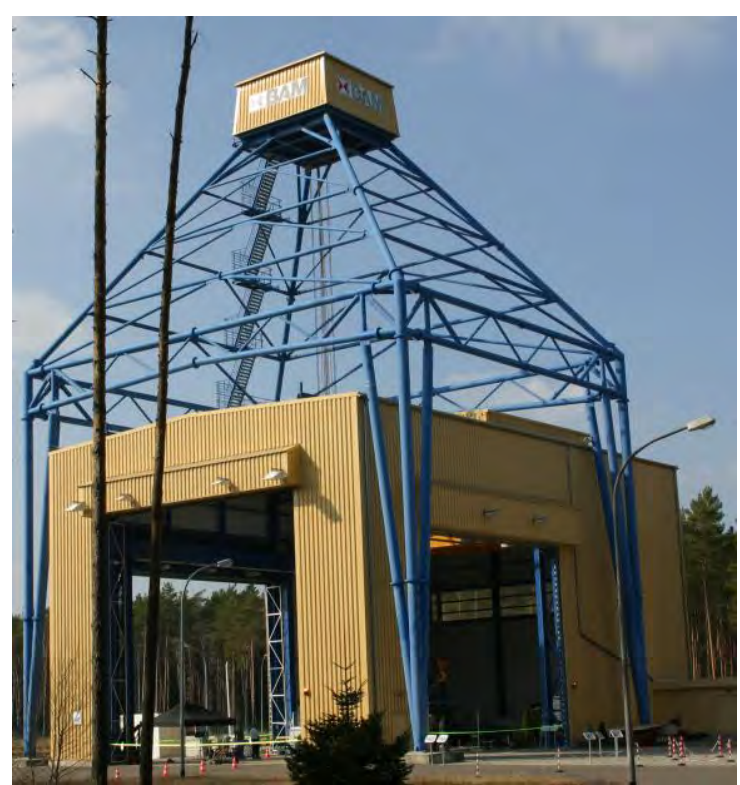

Fig. 1. BAM drop test facility

1) RAM Radioactive material 
For the moment the investigated experimental configuration covers a $0.4 \mathrm{~m}$ and a $5 \mathrm{~m}$ bottomside drop test as well as a $5.0 \mathrm{~m}$ long bottomedge drop test. The drop tests were performed onto an essentially unyielding target according to International Atomic Energy Agency (IAEA) Regulations [2]. The 200 metric tons BAM drop test facility is shown in Fig. 1. Fig. 2 gives an idea about container design and its global orientation before the $5 \mathrm{~m}$ long bottom-edge drop.

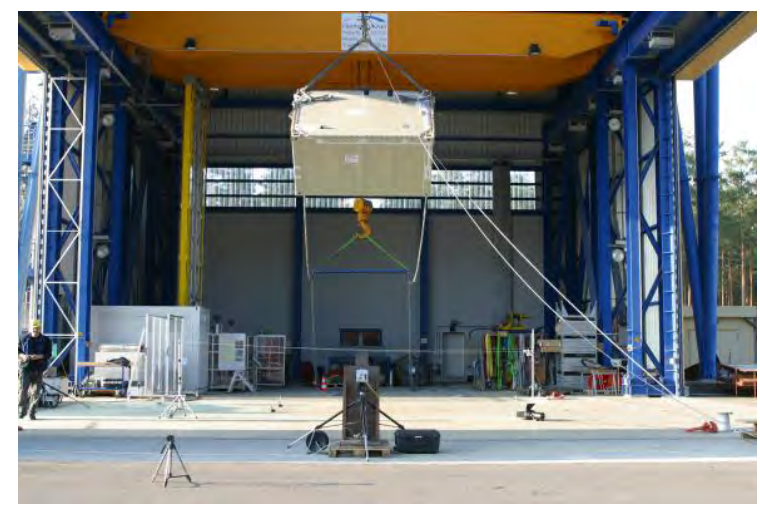

Fig. 2. Container before $5 \mathrm{~m}$ drop with the long bottom edge first onto the impact target

The container dimensions are $3.2 \mathrm{~m} \times 2.0 \mathrm{mx}$ $1.7 \mathrm{~m}$. The lid and bottom plates have a thickness of $5 \mathrm{~mm}$, whereas the side ones are $3 \mathrm{~mm}$ thick. The container mass is approximately $1,840 \mathrm{~kg}$.

\section{Numerical Simulations and Experimental Methods}

The simulations of the drop tests were carried out as fully dynamic FE calculations using the commercial code LS-DYNA $^{\mathrm{TM}}$ [3]. The FE model is shown schematically in Fig. 3.

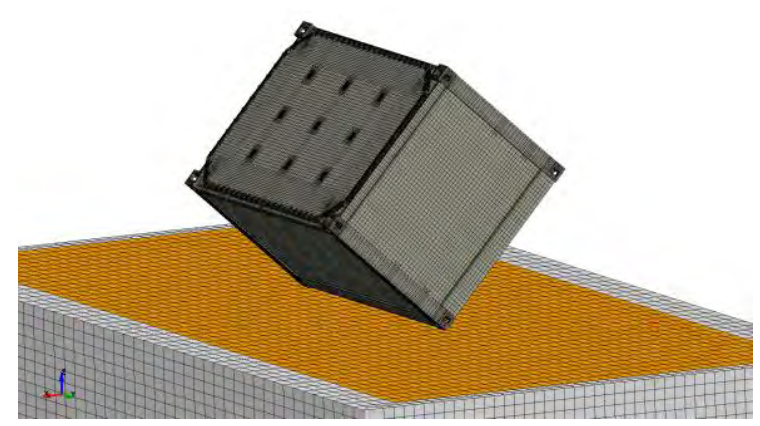

Fig. 3. FE model of container placed above the impact target
Due to the unsymmetrical construction of the steel sheet container and in order to facilitate also the analysis of complex drop scenarios without symmetry restrictions, the experimental set-up was simulated as full model. Fig. 4 depicts exemplarily the nodal displacements in ydirection and illustrates the plastic deformation of a small container side wall (no. 4a) $20 \mathrm{~ms}$ after first contact of the container with the foundation. This time interval has been selected owing to the fact that the inelastic deformation completely occurs within this impact time period.
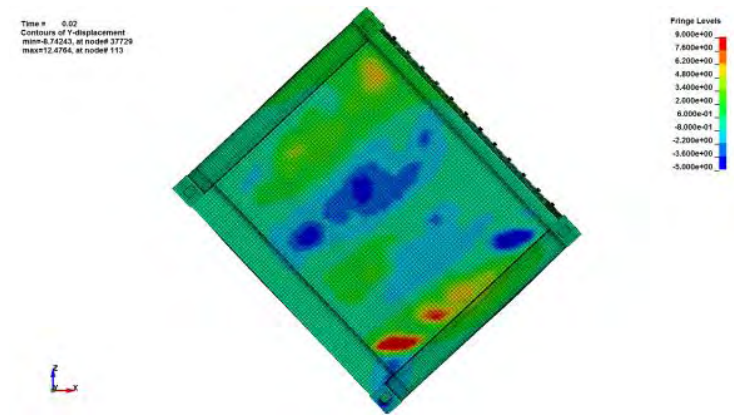

Fig. 4. Inelastic container sheet deformation simulated for the $5 \mathrm{~m}$ drop test with the long bottom edge first onto the target

The drop tests were monitored by a large number of simultaneously triggered biaxial electrical strain gauges and uniaxial accelerometers in order to measure local strains at selected positions and to determine the kinematic behaviour and orientation of the container during the impact. In addition to these standard sensor methods, two optical measurement principles have been involved. The Stereo Photogrammetry approach has been applied in a high-speed (HS) camera configuration to investigate the kinematic rigid body behaviour, relative local movements of the container or its structural parts in 3D, respectively. Furthermore, the complete 3D container shapes before and after the drop tests have been determined applying the optical measurement Fringes Projection Method. It was combined with Close Range Photogrammetry in this rather large volume to get the digital all around surface shape data with high precision [4]. Hence, further on the main focus is on qualification and data analysis of these optical measurement methods.

\section{D surface shape digitization}

The fringe projection method is in a sense already used as a standard approach for shape or deformation documentation and analysis in 
drop tests at BAM [4]. This full-field surface measurement method is applied before and after container deformation caused by drop impact. As a major result the real container surface shape initially defined by a 3D point cloud is mathematically represented using a dense meshed network of triangulated planes or spline functions. This approach provides you with a very flexible virtual measuring tool for $3 \mathrm{D}$ shape and deformation analysis. Using fringes projection method the container shape before and after drop has been determined. The deformation was calculated from the difference of these both states. Fig. 5 shows the inelastic remaining deformation of the same container side as presented in Fig. 4.

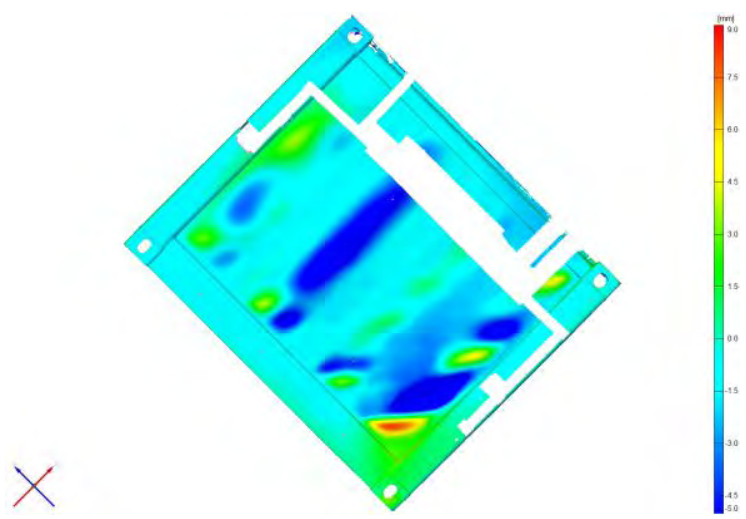

Fig. 5. Representation of a deformed container steel sheet wall after the $5 \mathrm{~m}$ drop

Fig. 5 shows the difference of normal displacement vector components scaled in accordance to deformation range in Fig. 4. A comparison between simulation and measurement results shows a good agreement. In both cases the container side buckled in a very similar way near the impacting lower ISO corners. Differences arise, however, necessarily because of the fact that the simulation is calculated with respect to ideally plane reference state before drop contact, where the optical digitization result refers to real metal sheet surface shape, respectively.

\section{Rigid body and dynamic deformation analy- sis}

Two calibrated synchronous triggered digital high-speed cameras have been used in the second $5 \mathrm{~m}$ drop test (Fig. 6). Stereo Photogrammetry used here is a measurement method based on contrasting points fixed on the surface under investigation. It provides 3D information about the dynamic behaviour of components expressed by coordinates, displace- ment, distance, velocity, acceleration and deformation as function of time.

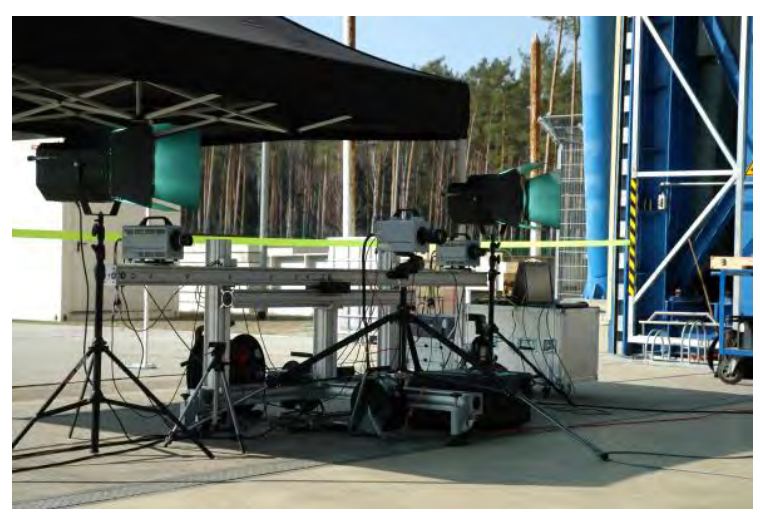

Fig. 6. Stereo camera setup used in the $5 \mathrm{~m}$ drop test with the long bottom edge first onto the target

The single camera visible in the centre of the image was given the task of documentation. Both PHOTRON FASTCAM-APX RS stereo cameras operated with a frame rate frequency of $10,000.0 \mathrm{~Hz}$ at a lateral resolution of 512 by 512 pixels approximately adapted to a $2.7 \mathrm{~m}$ by $2.7 \mathrm{~m}$ field of view (see Fig. 7).

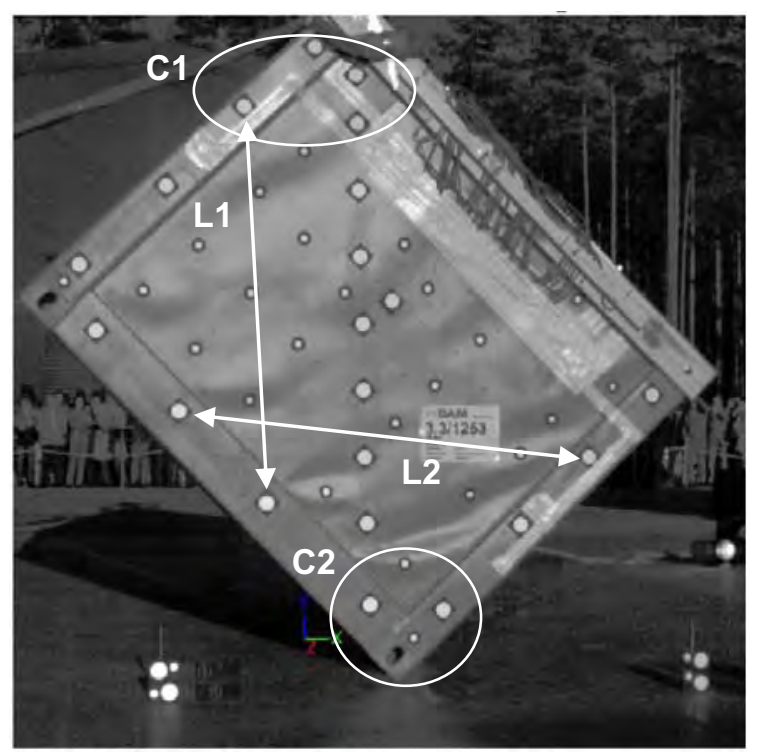

Fig. 7. Representation of a deformed steel sheet container with indicated point features: Distances between points (L1, L2) and point components $(C 1, C 2)$

The rigid body impact velocity and deceleration as well as dynamic and plastic deformation of container parts are important parameters of drop tests which can be experimentally determined. Fig. 8 shows the time dependent con- 
tainer height and velocity functions with respect to the container impact area.

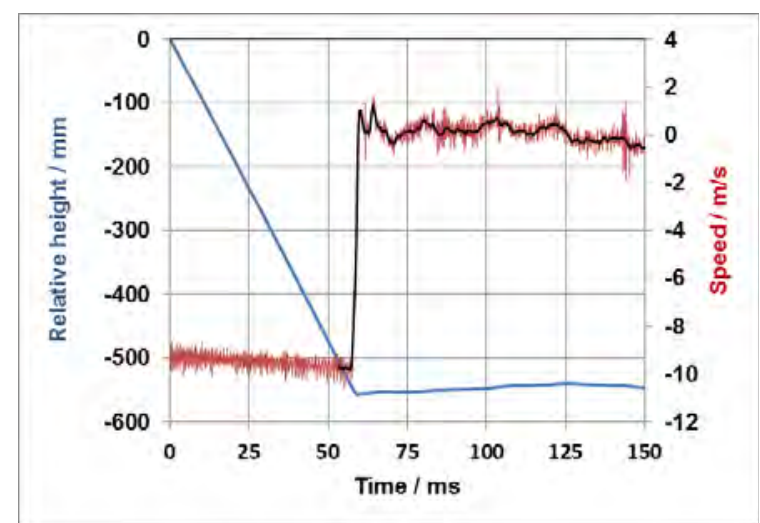

Fig. 8. Measured height data from stereo method together with directly calculated and approximated drop speed

The final container drop speed has been calculated from all speed data up to about $57.6 \mathrm{~ms}$ before impact using a linear fit function to be $-9.78 \pm 0.02 \mathrm{~m} / \mathrm{s}$. With respect to the pure principle of kinetic and potential energies conservation this experimentally defined value differs by $-1.4 \%$ from the ideal theoretical one. Checkups of camera image trigger signal and stereo setup length measure confirmed the high accuracy of frame rate (better than $1 \cdot 10^{-7}$ ) and the dimensional scale (better than $1 \cdot 10^{-4}$ ). Therefore, these effects could be excluded as technical reasons why differences between experimental and theoretical speed appeared. A further analysis has shown that the difference of this magnitude can be attributed to air drag.

Besides final impact velocity determination for energy balance purposes, the rigid body deceleration is another important parameter which can be experimentally determined in drop tests. It helps e.g. to estimate the maximum load on container or impact limiter components. In the experimental background discussed here the rigid body deceleration cannot be calculated directly from stereo images data because of unavoidable noisy velocity values derived from coordinates. An intermediate curve fitting step is necessary. Therefore, the height function has been fitted using piecewise non-parametric splines in a well-controlled manner. Characterizing the fit quality, a maximum height coordinate difference below $0.15 \mathrm{~mm}$ relative to the observed vertical movement of about $550 \mathrm{~mm}$ (see Fig. 8) was achieved. The speed function calculated from this and starting $57 \mathrm{~ms}$ before impact is also shown in Fig. 8. From this fit function the deceleration is derived. In Fig. 9 the results of two different experimental meth- ods are compared. The single axis acceleration sensor is manufactured by PCB Piezotronics Company. This sensor is sampled at a rate of $200 \mathrm{kHz}$, whereas the camera is based on a 10 $\mathrm{kHz}$ one.

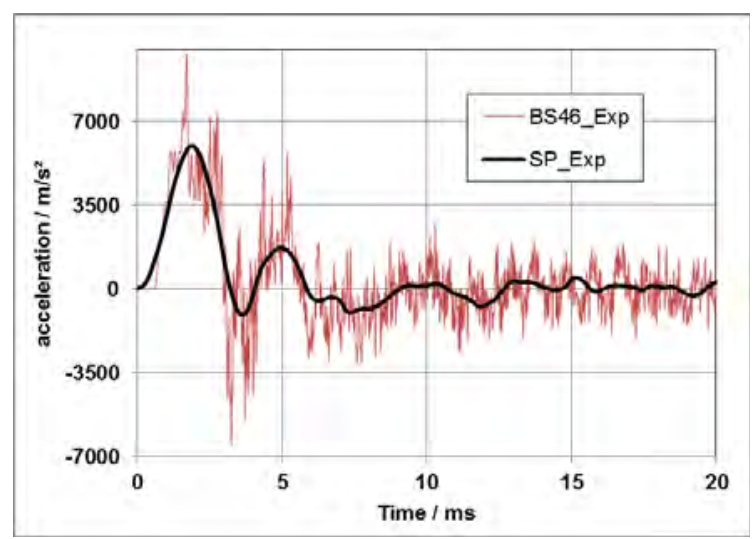

Fig. 9. Rigid body deceleration signals from piezoelectric accelerometer and Stereo Photogrammetry method

The low pass filtered sensor signal with cut-off frequency of $50 \mathrm{kHz}$ shows additional vibration components probably caused by natural sensor oscillations, metal sheet or structural container part vibrations. In total two accelerometers have been deployed mounted at both ends of upper traverse. The motion analysis results of Stereo Photogrammetry are based not on single points but small groups of points called point components (see components $\mathrm{C} 1$ and $\mathrm{C} 2$ in Fig. 7). This averaging approach has the advantage of noise reduction. The maximum deceleration value determined at the upper container position away from the contact area is $6000 \mathrm{~m} / \mathrm{s}^{2}$. Of course, the lower container part suffers from a harder mechanical impact with maximum deceleration level of about $7550 \mathrm{~m} / \mathrm{s}^{2}$. Due to the low spatial resolution the high speed Stereo Photogrammetry is especially suited to characterise rigid body kinematics in 3D. In this case, additional technical regulations or standards specifying the appropriate high frequency signal filtering approach are not necessary.

In addition to rigid body motion analysis Stereo Photogrammetry can effectively be applied to study elastic deformation. This provides another option to compare experimental and simulated data for even better FE model control. The vertical and horizontal container deformations explained by means of changes in point distances are presented in Fig. 10. 


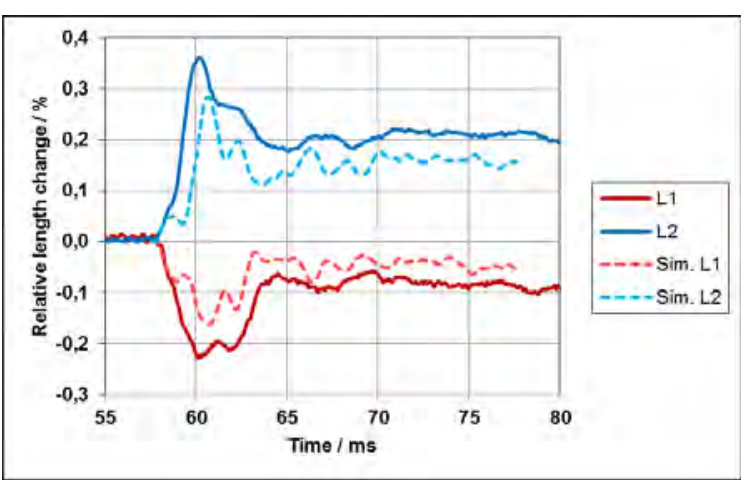

Fig. 10. Maximum elastic and remaining distance changes between surface points

Fig. 7 already depicts the defined distances L1 and L2 between selected container surface points. The magnitudes of L1 and L2 are approximately $1.5 \mathrm{~m}$. The coordinates of the selected surface points have been transferred from the stereo measuring system to the corresponding node positions in the FE model. A similar adjustment was made for the time scales. The time dependent changes of $\mathrm{L} 1$ and L2 tend to opposite directions due to the spacious container bending and compression effects. Changes in horizontal direction with a maximum at about $0.4 \%$ are higher by a factor of 2 compared with the vertical direction. Nearly the same conclusion is made from numerical simulation results regardless of the throughout slightly smaller values. In both approaches significant inelastic remaining deformation up to $0.2 \%$ can be detected.

\section{Conclusion and outlook}

High speed stereo photogrammetry and the fringes projection method have confirmed their suitability in harsh technical environment for 3D kinematic as well as dynamic and static deformation analysis in drop test scenarios, respectively. In particular, the full field surface shapes digitization method working in combination with common digital photogrammetry has become an experimental 3D method of choice with respect to both objectives, the required accurate experimental documentation and a flexible deformation analysis. In this respect, FE calculations play an important role for a better understanding of dynamic drop loads onto containers. Nevertheless, further work is still based to a high degree on comparison with experimental data. Numerical simulations will remain in special focus of the ongoing steel sheet container research project. Considering the results presented in this paper, the introduced optical measurement principles proved highly beneficial in order to validate the numerical simula- tions in addition to the standard sensor methods. The comparisons of the 3D surface shape digitization as wells as the dynamic deformation analysis with the results of the numerical calculations show that the FE model is suitable to describe the mechanical behaviour of the boxshaped container during drop tests. The findings reveal the necessity of further improvement of the numerical model. An important aspect to improve the FE calculation results is a more realistic container model. Instead of ideal plane walls made of steel sheets different in thickness the model should be based on the actual container surface shape. These data for the whole container body can be provided by the projected fringes method.

\section{References}

[1] Ellouz, M., Völzke, H., Protz, C.: "Container Safety Assessment for the Disposal of NonHeat Generating Radioactive Waste in the KONRAD Repository." INMM 53rd Annual Meeting, Orlando, Florida USA, July 15-19, 2012

[2] Müller, K., Quercetti, T., Melnik, N., Droste, B.: "Impact target characterisation of BAM drop test facility." Packaging, Transport, Storage and Security of Radioactive Material, Volume 19 (2008), Number 4, pp. 217-221, Maney Publishing, UK

[3] LS-DYNA Explicit Finite Element Code Version Is971d, Release 4.2.1, Revision 53450, Livermore Software Technology Corporation

[4] Gründer, K.-P., Kadoke, D., Müller, K., Musolff, A.: "Characterization of shock absorber deformation by optical surface digitization." Packaging, Transport, Storage and Security of Radioactive Material, Volume 19 (2008), Number 3, pp. 155-159, Maney Publishing, UK 